\title{
Research Article Hypergravity conditioning on ileal movements in rats
}

Mari Kimoto ${ }^{1}$, PhD; Jorge L Zeredo ${ }^{2,3}, D D S, P h D ; K a z u o ~ T o d a{ }^{1,3} D D S, P h D$

${ }^{1}$ Physiological Laboratories, Japan Women's University, Tokyo, Japan;

${ }^{2}$ University of Brasilia, Brasilia, Brazil, and ${ }^{3}$ Integrative Sensory Physiology, Graduate School of Biomedical Sciences, Nagasaki University, Nagasaki, Japan.

${ }^{*}$ Reprint requests to:

Prof. Kazuo Toda, DDS, PhD, Integrative Sensory Physiology, Graduate School of Biomedical Sciences, Nagasaki University, 1-7-1 Sakamoto, Nagasaki 852-8588, Japan.

Short Title: Hypergravity and ileal movements

Number of words in the Abstract: 250

Number of words in narrative text: 1879

Number of Figures: 4

Number of Tables: 0 


\section{Abstract}

INTRODUCTION: Stress is a common trigger for various physiological disturbances including feeding disorders. One of the possible mechanisms for feeding disorders may be linked to changes in intestinal movement caused by stress. Therefore, here we investigated in vitro stress-induced changes in ileal movement. METHODS: Rats (Wistar male, SPF) were divided into Control (1G) and hyperG groups. HyperG stress was applied daily for 10 min by a centrifugal apparatus for 1 to 30 days. Under barbiturate anesthesia, a $1 \mathrm{~cm}$-long section of the ileum was isolated and fixed in a Magnus-type chamber filled with tyrode solution. Intestinal movement was evoked by applying acetylcholine (Ach, $10^{-7}-10^{-5} \mathrm{~g} / \mathrm{ml}$ ). Antagonistic effects of adrenalin (Adr, $10^{-4}$ $\mathrm{g} / \mathrm{ml}$ ) on the Ach-evoked movements were also observed. RESULTS: Clear ileum movements were observed after Ach application. The movement pattern was phasic (early) and tonic (late). Peak amplitude of the phasic wave was dose-dependent on the Ach concentration. No significant differences in the averaged peak amplitude between control and hyperG groups were observed. The peak amplitude was decreased by Adr application in both control and hyperG groups; however, the degree of the decrement was higher in hyperG than in control at 1 day after stress loading. DISCUSSION: The present study indicates that gravity stress modified ileal movement. Although basic ileum movements evoked by Ach were not influenced by stress, they were modified by Adr at a quite early stage after stress loading, suggesting an increase in the sensitivity of Adr receptors but not of Ach receptors in the ileum.

Keywords: Hypergravity, ileum, intestinal movement, stress, rat 


\section{Introduction}

Stress is a common trigger for various physiological disturbances including feeding disorders (13). It is especially important to acknowledge the close relationship between stress and digestive function (4). The most common physiological disorder underlying the digestive problems is reported to be stress $(1,21)$. As a result of stress, feeding disorders, and the consequent increase or decrease in body weight, have been reported in both humans and experimental animals $(7,11,15)$. Stress affects the gastrointestinal tract function as a part of the visceral response $(16,22)$. Various stressors are reported to induce similar profiles of gut motor-functional alterations, including inhibition of gastric emptying, increase or decrease of intestinal movement including segmentation, pendulation and peristalisis, and hypersensitivity to colorectal distension (8). These disorders may be linked to changes in intestinal movement, which modulate total gastric function or are modulated reciprocally by specific gastric functions (12).

Intestinal movement is modulated by neurotransmitters released from nerve terminals in the autonomic nervous system (and the adrenal medulla): acetylcholine stimulates and adrenalin/noradrenalin inhibits smooth muscle contraction (6). Generally, autonomic structures both in the central and in the peripheral nervous system are sensitive to stress. The hypothalamus in the central nervous system, and the adrenergic sympathetic and cholinergic parasympathetic target organs in the peripheral nervous system are readily affected by stressful stimulation (14). In addition, it is conceivable that changes in the sensitivity of target organs to the autonomic neurotransmitters could also be modified by stress. To clarify this, we investigated stress-induced changes in ileal movement.

Hypergravity, as obtained through centrifugation, has been proposed as a means to countermeasure the effects of long-time exposure to microgravity in future manned missions to other planets. Hypergravity could also be used to enhance the effects of force-training in athletes. However, careful consideration on possible side-effects of centrifugation on all bodily functions and systems should be carried out before it can be used in a regular basis. Previous animal studies have shown general effects of hypergravity in the body, such as changes in body weight and gustatory sensation in rats $(9,10)$.

Hypergravity may modify the normal body physiology through a stress response. In rats, hypergravity through centrifugation has been found to induce changes similar to those of other well-known stressors. 


\section{Methods}

\section{Animals}

The methods described here follow the ethical guidelines and received approval by the Animal Welfare Committees of Japan Women's University and Nagasaki University.

Forty eight rats (Wistar male, SPF, body weight 144-219 g) were divided into Control (1G) and hyperG groups. Each group was further divided in 1 day, 15 days, and 30 days groups ( $n=8$, each). The animals were kept in a colony room next to the centrifuge room. 


\section{Procedure}

HyperG stress was applied every day at 10:00 AM for 10 min during 30 days. The animals were moved in pairs from the colony room to the centrifuge room and loaded into the centrifuge. A centrifugal apparatus (H26-F, Kokusan, Tokyo, Japan) was used, as described elsewhere (10). The arm length of the centrifuge was $15 \mathrm{~cm}$, and to produce hyperG, the speed of rotation was set to $108 \mathrm{rpm}$ (resulting in an acceleration vector of $2.2 \mathrm{G}$ ). During loading, rats were placed with head-central orientation in the mesh case at the end of the centrifuge's arm (gravity stress was thus a gradient, with the most $g$ force being at the tail end of the rat). The mesh case was freely-rotating so that the vector of force was always perpendicular to the case floor. In the control group, the rats were placed in the centrifuge but not rotated. At the end of the centrifugation the animals were either returned to the colony room or anesthetized for experimentation. Experiments were done at 1,15 and 30 days of gravity-loading.

Under barbiturate anesthesia (Nembutal, $20 \mathrm{mg} / \mathrm{kg}$, i.p.), a $1 \mathrm{~cm}$-long portion of the ileum was isolated. After removal of the tissue sample, and while still under surgical anesthesia, the animal was killed by cervical dislocation. The inner contents of the isolated ileum were washed away with Tyrode solution. After that, the isolated ileum was fixed into a Magnus-type chamber filled with Tyrode solution (temperature: $37^{\circ} \mathrm{C}$ ). The proximal end of the preparation was set upward and connected to a strain gauge through a cotton thread (Daruma \#30, Yokoi, Osaka, Japan). The distal end of the preparation was fixed to the bottom of the chamber (Fig 1, upper panel).

Ileal movements were evoked by applying acetylcholine (Ach, $10^{-7}-10^{-5} \mathrm{~g} / \mathrm{ml}$ ). Antagonistic effects of adrenalin (Adr, $10^{-4} \mathrm{~g} / \mathrm{ml}$ ) on the Ach-evoked movements were also observed. These solutions $(0.3 \mathrm{ml})$ were topically applied onto the ileum preparation using an injection syringe. lleal movements were estimated by the force of contraction as captured by a strain-gage, whose signal was amplified (x100) and recorded continuously on a pen-recorder (8M-15,OMNICORDER, SANEI, Tokyo, Japan).

The magnitudes of the Ach-evoked ileal movements were calculated as the maximum amplitude of the phasic contraction subtracted from amplitude of the base line contraction (Fig.1, lower panel, arrow A). The antagonizing effect of Adr on the Ach-evoked response was estimated by the amplitudes from the baseline contraction (Fig.1, lower panel, arrow B).

\section{Statistical analysis}

Significant differences were determined by ANOVA followed by post-hoc Bonferroni test. $P$ values of less than .05 were considered statistically significant. All data are 
expressed as means \pm SEM.

\section{Results}

[Fig. 1 here]

[Fig. 2 here]

Clear peristaltic movements were observed after Ach application. The movement pattern was in a phasic (early) and tonic (late) fashion as shown in Fig. 1. Peak amplitude of the phasic wave was dose-dependent on the Ach concentration. Fig.2 summarizes the dose-response curves of Ach-induced ileal movements. Open and closed circles show the amplitude of contraction after Ach application in the chamber in control and hyperG groups, respectively. No significant differences in the peak amplitude of contraction evoked by Ach were observed between control and hyperG groups.

Ach-induced ileal movement was antagonized by Adr. Fig.3 shows typical examples of Adr effects on the Ach-induced contraction 1 day after hyperG loading. Upper trace shows Adr-antagonization against $10^{-6}$ Ach contraction. (A) shows baseline contraction level before Ach application. Phasic (B) and tonic contractions were induced, and during tonic contraction, application of Adr reduced both tonic and baseline contractions. This inhibition lasted until the whole preparation was rinsed with Tyrode solution.

Lower trace shows Adr-antagonized effects on the $10^{-5}$ Ach-induced contraction. In this case, although Ach-induced contraction was inhibited by Adr, the magnitude of the effect was lower as compared with the $10^{-6}$ Ach-induced contraction.

[Fig. 3 here]

[Fig. 4 here]

Fig. 4 shows a summary of Adr effects on the Ach-induced contraction in control and hyperG-loading groups. The initial amplitude was estimated by the contraction height between the amplitudes of baseline and of phasic contraction. Amplitudes of contraction 10, 30 and $60 \mathrm{~s}$ after Adr application were measured. (Top graph) shows the Adr effect 1 day after hyperG-loading. As compared with control (open circle), Adr well antagonized the Ach-induced contraction. Especially, at 10 and $30 \mathrm{~s}$ after Adr application, a significant decrease in amplitude was induced, with $F(1,14)=5.52, p=.03$ and $F(1,14)=4.73, p=.04$, respectively. However, after 15 and 30 days of loading, there was no significant difference in response amplitude between control and hyperG groups. 


\section{Discussion}

Digestive smooth muscles are innervated by autonomic nerve fibers of the sympathetic and parasympathetic divisions. The former provides excitatory cholinergic innervation and the latter provides inhibitory adrenergic innervation. lleal movements are neurally regulated in vivo by the autonomic nervous system (6). However, in the present study, an in vitro preparation was isolated without direct innervation, therefore, instead of nerve stimulation, Ach or Adr was used to stimulate pharmacologically ileal smooth muscle.

The present study indicates that gravity-stress modified ileal movement in vitro. Although the high-speed rotation of the centrifuge may have played a significant role as well; therefore, these two forces cannot be dissociated in this study. Peristaltic movements by Ach were not influenced by stress; however, they were modified by Adr at an early stage ( 1 day) after gravity loading.

\section{Changes in ileum Ach-sensitivity induced by stress}

Ach receptors (cholinergic receptors) are classified into two main pharmacological types: Ionotropic nicotinic acetylcholine-receptors and metabotropic muscarinic acetylcholine-receptors (17). In the smooth muscles of the ileum, muscarinic acetylcholine receptors are activated by Ach released by parasympathetic nerve terminals. In the present study, no significant differences were found in the Ach-induced ileum movements between control and hyperG groups. Therefore, basic Ach-sensitivity was not influenced by gravity-stress. In addition, it is generally accepted that the basic tone of the ileum is sustained by Ach (2). Thus, it is presumed that the smooth muscle-tone in the ileum was not changed by stress-loading. Because the present study was performed in vitro, and the effects of stress-loading on ileum function had a peripheral origin, probably changes in Ach-sensitivity and/or subcellular mechanisms of ileum smooth muscles that were induced in the whole animal still remained after preparation in vitro (2).

Several studies indicate that the expression of Ach receptors, both muscarinic and nicotinic, are modified under various conditions, such as chronic pain, general anesthesia, and muscle tension (19). However, as shown in this study, gravity-stress did not influence Ach sensitivity in the ileum.

\section{Changes in ileum Adr-sensitivity induced by stress}

In contrast to Ach sensitivity, the antagonizing effects of Adr on the Ach-induced 
contraction was observed after 1 day of stress-loading. At 15 and 30 days, no significant Adr-antagonizing effects were detected.

The body's homeostasis is constantly challenged by stressors. Under stressful conditions, homeostasis is re-established by various physiological endocrinological and behavioral adaptive responses (13). In short-term stress, neuronal responses are recruited against stressors, however, during long-term stress, neuroendocrine hormones play a major role in the regulation of both basal homeostasis and responses against stress (18). These stress responses are mediated by the endogenous stress system, located both in the central nervous system and in peripheral organs. Close interaction between central and peripheral components are critical $(5,20)$.

In the present study, gravity stress was applied on the whole animal and peristaltic movement was recorded in vitro just after the ileum preparation was isolated. Therefore, changes in sensitivity to Adr were induced by central and peripheral components of the stress system. However, during recordings, because central components were removed by the in vitro preparation, only peripheral mechanisms were studied. One of the possible mechanisms for our observations could have been an increase in the number of adrenergic receptors at the neuromuscular junction in the ileum, resulting in increased Adr sensitivity after gravity-stress in the ileum. This change was observed after only 1 day of stress-loading; therefore, it is presumed that this change was predominantly mediated by rapid neuronal mechanisms, rather than slow humoral mechanisms; nevertheless, we cannot rule out the alternative possibility of the change in Adr sensitivity to be a secondary adaptation to the autonomic regulation of blood flow in the abdominal cavity (3). At 15 and 30 days, the fact that there were no significant differences in Adr sensitivity was probably due to re-organized new homeostasis, including humoral components. It should be noted that due to technical limitations, namely the short-arm centrifuge, the rotation speed was high and the effects observed here may be at least in part attributed to rotation, and not exclusively to hyperG loading.

In conclusion, gravity-stress modified ileal movements. Basic ileum movements were not influenced by stress; however, they were modified by Adr after stress-loading, suggesting an increase in the sensitivity of Adr receptors but not of Ach receptors in the ileum. Hypergravity and high-speed centrifugation should be considered with caution for the space program, particularly in regards to the autonomic regulation of the digestive function. 


\section{Acknowledgements}

The authors thank Mutuko Kaku, Yukie Saitou, Mana Hayashida, Makiko Andou,

Satomi Hayashida, Yuki Miyazawa and Norie Yamazaki (Japan Women's University) for their skilful assistance. 


\section{References}

1. Adam TC, Epel ES. Stress, eating and the reward system. Physiol Behav. 2007;91:449-58.

2. Cohen ML, Landry AS. Vasoactive intestinal polypeptide: increased tone, enhancement of acetylcholine release, and stimulation of adenylate cyclase in intestinal smooth muscle. Life Sci. 1980;26:811-22.

3. Convertino VA. Mechanisms of blood pressure regulation that differ in men repeatedly exposed to high-G acceleration. Am J Physiol Regul Integr Comp Physiol. 2001;280:R947-58.

4. Dallman MF. Stress-induced obesity and the emotional nervous system. Trends Endocrinol Metab. 2010;21:159-65.

5. Eldar S, Ricon T, Bar-Haim Y. Plasticity in attention: implications for stress response in children. Behav Res Ther. 2008;46:450-61.

6. Feldberg WS. On the origin and function of the acetylcholine in the intestinal wall. Proc R Soc Lond B Biol Sci. 1950;137:285-92.

7. Greeno CG, Wing RR. Stress-induced eating. Psychol Bull. 1994;115:444-64.

8. Kadlec $\mathrm{O}$, Horacek J. Inhibition of peristaltic activity in the guinea-pig ileum by specific stress stimulus; its reversal by naloxone and indomethacin. Life Sci. 1980;27:1557-62.

9. Kimoto M, Takase S, Shomure E, Suzuki A, Zeredo JL, et al. High gravity modifies feed efficiencies and chemical sensation in rats. Abstract Autonom Neurosc. 2005;119:118-18.

10. Kimoto M, Zeredo JL, Toda K. Irritant-drinking behavior can be modulated by gravity-stress loaded in developing but not in adult rats. Stress \& Health. 2011; 27:34-41.

11. Kyrou I, Tsigos C. Stress hormones: physiological stress and regulation of metabolism. Curr Opin Pharmacol. 2009;9:787-93.

12. Larauche M, Kiank C, Tache Y. Corticotropin releasing factor signaling in colon and ileum: regulation by stress and pathophysiological implications. J Physiol Pharmacol. 2009;60 Suppl 7:33-46.

13. Leidy NK. A physiological analysis of stress and chronic illness. J Adv Nurs. 1989;14:868-76.

14. Lo Sauro C, Ravaldi C, Cabras PL, Faravelli C, Ricca V. Stress, hypothalamic-pituitary-adrenal axis and eating disorders. Neuropsychobiology. 2008;57:95-115. 
15. Martin MS, Martin F, Lambert R. Adaptation of restraint stress to body weight in the rat. Digestion. 1969;2:253-61.

16. Mazzon E, Sturniolo GC, Puzzolo D, Frisina N, Fries W. Effect of stress on the paracellular barrier in the rat ileum. Gut. 2002;51:507-13.

17. McCarthy MP, Earnest JP, Young EF, Choe S, Stroud RM. The molecular neurobiology of the acetylcholine receptor. Annu Rev Neurosci. 1986;9:383-413.

18. McEwen BS. Physiology and neurobiology of stress and adaptation: central role of the brain. Physiol Rev. 2007;87:873-904.

19. Namimatsu A, Go K, Ohara H, Yoneda R. Changes in muscarinic acetylcholine receptors in the isolated duodenum from repeatedly cold-stressed rats and the effect of neurotropin. Life Sci. 1992;50:1993-2000.

20. Romeo RD, Bellani R, Karatsoreos IN, Chhua N, Vernov M, et al. Stress history and pubertal development interact to shape hypothalamic-pituitary-adrenal axis plasticity. Endocrinology. 2006;147:1664-74.

21. Torres SJ, Nowson CA. Relationship between stress, eating behavior, and obesity. Nutrition. 2007;23:887-94.

22. Vicario M, Guilarte M, Alonso C, Yang P, Martinez C, et al. Chronological assessment of mast cell-mediated gut dysfunction and mucosal inflammation in a rat model of chronic psychosocial stress. Brain Behav Immun. 2010;24:1166-75. 


\section{Figure legends}

Fig.1. Diagram of the experimental setting with a typical example of Ach-evoked ileum movement and antagonizing effect of Adr. (A) Ach evoked a phasic-tonic response, with the tonic part sustaining the response's maximum amplitude. (B) After Adr application, the tonic component of the response was markedly diminished.

Fig. 2. Dose-response relationship between amplitudes of Ach-evoked tonic response (ordinate) and Ach concentration (abscissa) at 1, 15 and 30 days after stress-loading. Values indicate means \pm SEM.

Fig. 3. A typical example of Adr antagonization on the Ach-evoked contraction. (A) tonic contraction before Ach application, (B) Ach-evoked contraction, (C) Adr antagonization. 1: Ach application 2: just before Adr application, 3: rinse. Note that at $10^{-6}$ $\mathrm{g} / \mathrm{ml}$ Ach concentration, Adr strongly antagonized tonic contraction, but at $10^{-5} \mathrm{~g} / \mathrm{ml}$ concentration, this did not occur.

Fig. 4. Antagonizing effect of Adr on the Ach-evoked contraction at 1, 15 and 30 days after stress-loading. Ordinate: amplitude of the ileum contraction. Negative values show decrease in amplitude below tonic contraction. Abscissa: time after Adr application. 10, 20 and $60 \mathrm{~s}$ correspond to the time scale in the Fig.3. Values indicate means $\pm S E M$. Asterisks denote statistically significant difference $(p<0.05)$. 
Figure4Ach $10^{-6} \mathrm{~g} / \mathrm{mL}$ - 1 st day
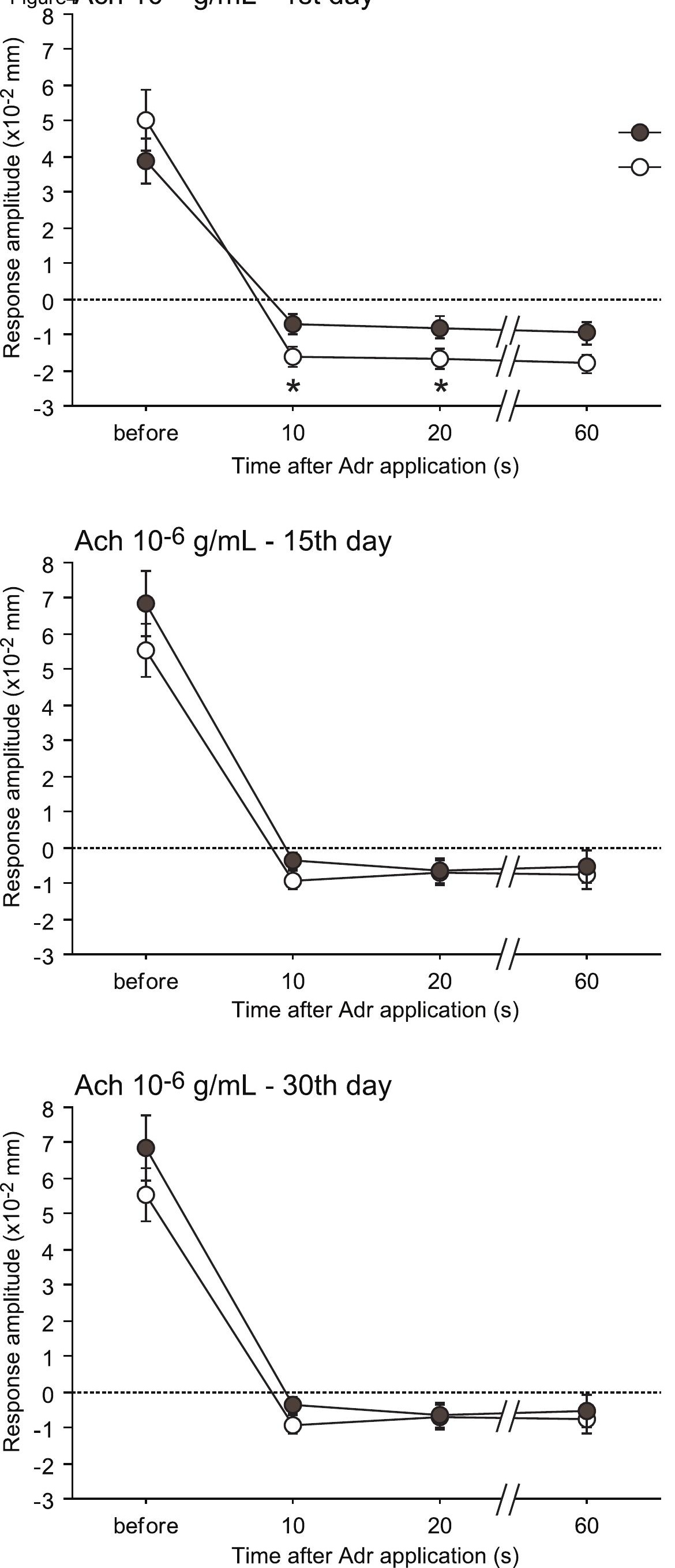